\title{
Proprioception-Based Grasping for Unknown Objects Using a Series-Elastic-Actuated Gripper*
}

\author{
Tianjian Chen $^{1}$ and Matei Ciocarlie ${ }^{1}$
}

\begin{abstract}
Grasping unknown objects has been an active research topic for decades. Approaches range from using various sensors (e.g. vision, tactile) to gain information about the object, to building passively compliant hands that react appropriately to contacts. In this paper, we focus on grasping unknown objects using proprioception (the combination of joint position and torque sensing). Our hypothesis is that proprioception alone can be the basis for versatile performance, including multiple types of grasps for objects with multiple shapes and sizes, and transitions between grasps. Using a series-elastic-actuated gripper, we propose a method for performing stable fingertip grasps for unknown objects with unknown contacts, formulated as multi-input-multi-output (MIMO) control. We also show that the proprioceptive gripper can perform enveloping grasps, as well as the transition from fingertip grasps to enveloping grasps.
\end{abstract}

\section{INTRODUCTION}

Proprioception, or the ability to perceive the relative positioning of neighboring body parts as well as the muscle effort deployed to produce it, is a fundamental human sense. Together with vision and tactile sensing, it plays a unique and important role in human hand perception [1]. For robotic manipulation, proprioception is translated as the combination of joint position and torque sensing (assuming a hand comprised exclusively of revolute joints).

Compared to vision and tactile sensing, both of which have been studied extensively in the context of manipulation, we believe that grasping with proprioception is still an important area to advance. On one hand, vision and tactile sensing have intrinsic limitations, such as occlusion for vision and hardware complexity for tactile sensing. On the other hand, when all these senses are available, they can still complement each other. Demonstrating manipulation capabilities based exclusively on proprioception becomes a useful exercise: we believe the more a hand can do with only one sensing modality, the more versatile it will be when multi-modal sensory information gets integrated.

As we show here, proprioception is promising in providing the hand with the ability to adapt to the previously unknown shape of the object, and to execute stable grasps. We note that there are multiple ways for hands to adapt to an object: while fully-actuated hands use sensor information (such as proprioception here) to perform active adaptation, underactuated hands are good examples of passive adaptation without the use of sensing. However, the former have the advantage of

\footnotetext{
*This work was supported in part by the NASA Early Space Innovations program through award NNX16AD13G.

${ }^{1}$ Both authors are with the department of Mechanical Engineering, Columbia University, New York, NY 10027, USA. \{tc2764, matei.ciocarlie\}@columbia.edu
}

versatility: proprioceptive grippers can provide compliance similar to passive mechanisms, but can also change behavior at runtime and selectively execute different types of grasps. Of course, the price paid for the additional versatility is the increased complexity of the sensory setup.

In this study, we explore the problem of grasping using only proprioceptive feedback, without any contact information or knowledge of object pose and properties. To the best of our knowledge, we are the first to show that a robot hand can perform all the following tasks using proprioception exclusively:

- execution of fingertip grasps for unknown objects;

- execution of enveloping grasps for unknown objects;

- on-demand transitions between fingertip and enveloping grasps.

Our main contributions are to provide methods for the tasks above, and, in the process, demonstrate their effectiveness by experiment. Our results indicate that the proprioceptive gripper is more versatile in the range of fingertip grasps it can perform, compared to our two baselines: an emulated underactuated gripper commanding fixed torques to the joints, as well as a physically constructed underactuated gripper. In addition, our gripper also displays the ability to execute enveloping grasps and to transition to them from fingertip grasps. Both examples of increased versatility were achieved using proprioception as the only available sensing modality.

\section{RELATED WORK}

Researchers have been exploring real-time sensing and control as an alternative to vision-based planning in manipulation. Assuming object information is available, modelbased controllers can perform grasping or in-hand manipulation. For example, Yoshikawa et al. presented studies (e.g. [2]) on hybrid force-position control for manipulation. Arimoto et al. [3] derived the dynamics of a dual-finger gripper and proposed a controller which can regulate the object position and orientation. Caccavale et al. [4] proposed an impedance controller to keep track of desired object trajectory and ensure the grasp quality simultaneously. Unlike our approach, these methods require complete information of the hand-object system.

When the models of the objects are not available, researchers either relied on assumption about the contacts, or used sensor-based techniques for grasping. For example, Schneider and Cannon [5] studied object impedance control using multiple manipulators. Arimoto et al. [6] and Yoshida et al. [7] studied "blind grasping" using two fingertips. However, these studies assume the contacts only happen at 


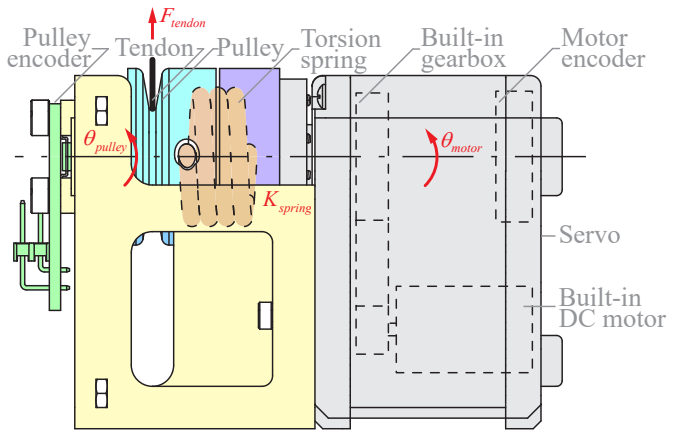

Fig. 1. SEA module.

the end points or the end hemispheres of the fingertips. Wang et al. [8] proposed a controller that can search appropriate finger contact locations using haptic feedback and can switch between control modes for different surfaces. Platt et al. [9] presented a study on changing the contact configuration by following the gradient of grasping objective functions using six-axis loadcell data. Hsiao et al. [10] proposed a contactreactive method using tactile sensing to deal with uncertainty. However, these methods require contact sensing methods, such as tactile sensors or in-finger load cells. In contrast, our approach does not make any assumptions about contact location or state, and does not require tactile sensing data.

Torque measurement is often used for grasp control. Researchers have developed several robotic hands with force or torque sensing. For example, the Robonaut Hand [11] and the DLR Hand II [12] have strain gauges or force-torque sensors embedded in their fingers. The hand of the DOMO robot [13] and the hand of the Obrero robotic platform [14] make use of the Series Elastic Actuators (SEA), which are a type of actuators with elastic components in series with the motor to sense the torque [15]. Furthermore, the DLR HandArm System [16] incorporates the Variable Impedance Actuators, which are SEAs whose spring stiffnesses are actively controlled. These hardware designs offer high performance, but at the cost of high complexity and large overall packages.

As an alternative, researchers have developed underactuated hands that do not require sophisticated sensing and control, and this types of hands are good baselines to compare against. Underactuated hands can adapt to the object and make a grasp by the virtue of carefully-designed torque ratios between joints. The Harvard Hand [17], iHY Hand [18], Robotiq Hand [19], and Velo Gripper [20] are good examples in this category. However, even though underactuation simplifies control, it generally does not provide as much dexterity as full actuation. Many of the hands above can only perform certain types of grasps, or lack the flexibility to choose the configuration after making the grasp.

\section{HARDWARE PlATFORM}

While joint position sensing is ubiquitous for fullyactuated robot hands, torque sensing and control is not common in commercially available manipulators. This compelled us to design our own hardware testbed. We implemented torque sensing and control with Series Elastic Actuators

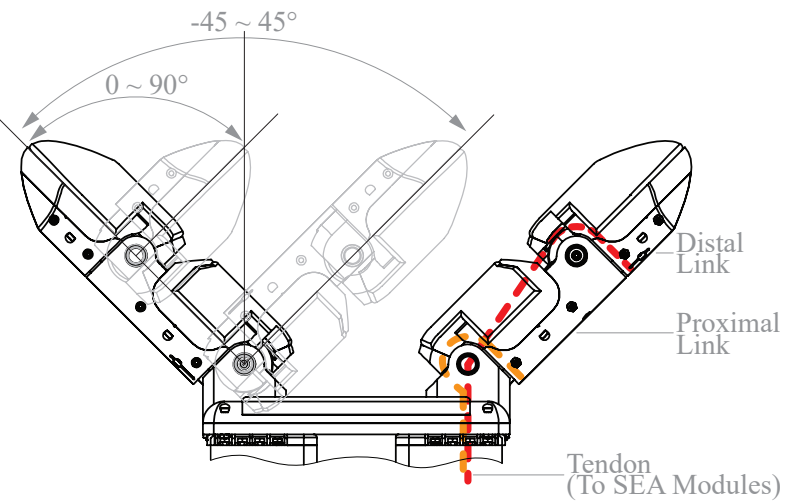

Fig. 2. Schematics of the gripper.

(SEA), a method known for high-fidelity torque control, shock protection, and human-safety [15].

1) SEA Module: Similar to the design from Ates et al. [21], we developed a simple and compact SEA module (Fig. 11. A position-driven servo (gray) is used as the driving motor, which receives position commands and returns the current position (measured by the built-in potentiometer), i.e., $\theta_{\text {motor }}$ can be measured. A torsion spring (orange) is used as the elastic component, connecting the motor shaft (purple) and the pulley shaft (blue). A Spectra cable is tied on the pulley to transmit the force to the finger joint. An absolute magnetic encoder (in green) is mounted on the end of the pulley shaft to measure $\theta_{\text {pulley }}$. In steady-state, the force in the tendon can be calculated as the product of spring stiffness and deflection divided by pulley radius:

$$
F_{\text {tendon }}=K_{\text {spring }} \cdot\left(\theta_{\text {pulley }}-\theta_{\text {motor }}\right) / R_{\text {pulley }}
$$

2) Gripper Design: The gripper consists of two fingers and each finger has two links, shown in Fig. 2 In each joint, flexion is powered by the tendon (shown as colored dash lines in Fig. 2p connected to the SEA pulley, and the extension is driven by a restoring spring. The tendon connected to the distal joint (the red dash line) goes right through the axis of the proximal joint so that the torques of proximal and distal joints are fully decoupled.

3) SEA-level Control: There are three SEA-level control modes: motor position control, pulley position control and torque control, and they can be switched online. We note that we built joint-level or hand-level controllers on top of these SEA-level controllers. For example, joint position and torque control can be achieved by SEA pulley position and torque control with a simple linear conversion.

\section{FINGERTIP GRASPING}

Fingertip grasps commonly refer to grasps where only the most distal links of each finger make contact with the object. We note that it does not necessarily mean the contacts are located in the very end of the fingers, so contact locations are still unknown. This type of grasp is important not only for precision tasks, but also for cases where a more stable enveloping grasp is not immediately available because of the environment (an object laying on a table, against a backdrop, 


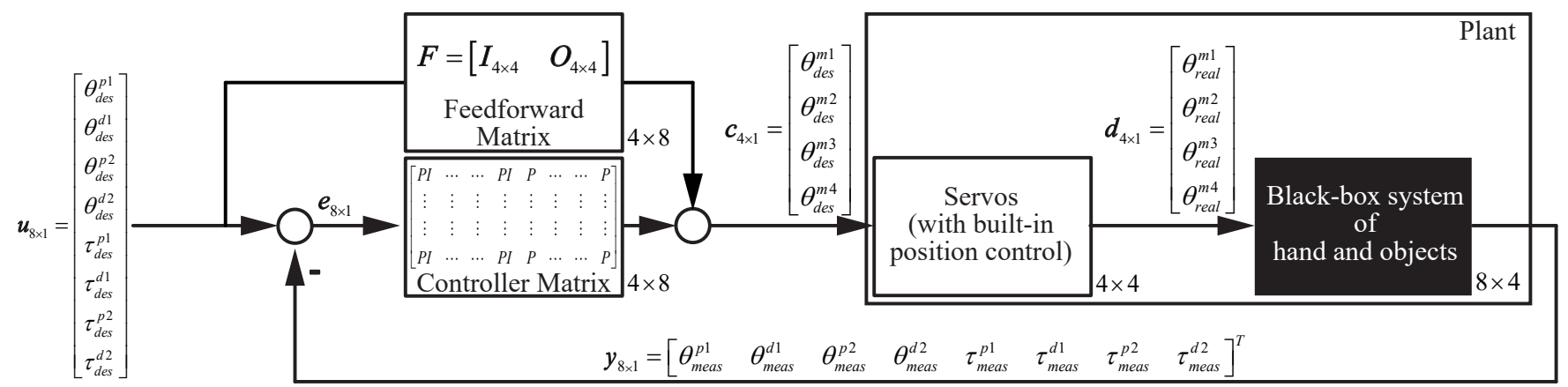

Fig. 3. Schematics of the MIMO Grasping Controller. (Subscripts: des—desired value, meas—measured value, real—real value. Superscripts: $\mathrm{m} —$ motor, p-proximal link, d-distal link).

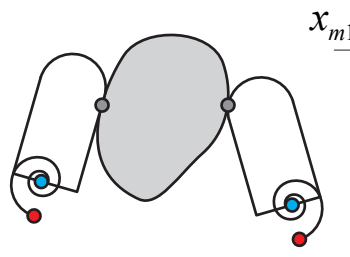

(a) Grasping with two fingers

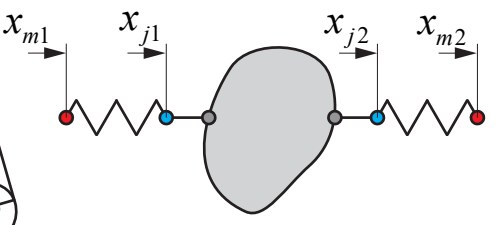

(b) Linear spring analogy

Fig. 4. Problem illustration.

etc.). In this section, we introduce a control algorithm which can perform stable fingertip grasps for unknown objects.

\section{A. Problem Statement}

The objective of our algorithm is to increase the torques applied at the joints (and implicitly the contact forces) in a "stable" fashion after making initial contacts. In other words, we need to find an increase in joint torques that produces no net wrench on the object. For a hand with multi-link fingers, this is not straightforward given that the joint torques need to be coordinated in the absence of information on object shape and contact locations. It is necessary to note that we are not solving contact planning problem, and we wish to develop reactive control strategies that do not require pre-planning.

Our insight is that proprioception alone can characterize grasp stability. For an SEA-powered proprioceptive gripper, an unbalanced net wrench will produce object movement against the compliant elements, which we can measure. Therefore, we formulate the goal of grasp stability as the one of minimizing object movement while applying forces. Since we do not have a direct measure of object movement in Cartesian coordinates by using only proprioception, we use the change of joint position in joint space (measured by SEA) during grasping as a proxy for object movement.

For intuition, consider the analogies shown in Fig. 4 , Figure (a) shows a (simplified) scenario in which the motors (red dots) are driving the torsion springs, and the springs are pushing the fingers (blue dots) to make a grasp (gray dots). (b) shows a simpler one-dimensional abstraction using linear springs, with similar color-coding as in (a). Here, we actively control the positions $x_{m 1}$ and $x_{m 2}$ (which translate to motor positions $\theta_{\text {motor }}$ on the real gripper) to apply forces, and measure $x_{j 1}$ and $x_{j 2}$ (which translate to pulley positions

$\theta_{\text {pulley }}$ linearly mapped to joint positions). We aim to keep $x_{j 1}$ and $x_{j 2}$ constant as we squeeze.

\section{B. MIMO Grasping Controller}

Our key insight is that the problem of grasping unknown objects can be solved even in the absence of contact information, by using proprioception as inputs for a multiinput-multi-output (MIMO) proportional-integral (PI) control. Without knowledge of object geometry, contact locations and contact states, it is impossible to fully model the dynamic system analytically. However, a proprioceptive platform still provides sensory access to the variables that characterize the grasp stability, and the PI control framework provides ways to regulate these variables even though the analytical relationship is not constructed. We thus aim to use a feedback scheme operating exclusively in the sensory space of the robot, without explicitly modeling the physics of the gripper and the object.

Fig. 3 shows the block diagram of the MIMO control loop. Here, the controller is constructed on top of the lowlevel sensing, so we consider the joint angles and torques are already obtained from SEA measurements. The reference vector $\boldsymbol{u}$ consists of desired joint angle values $\left(\theta_{d e s}^{p 1}, \theta_{d e s}^{d 1}\right.$, $\theta_{d e s}^{p 2}, \theta_{d e s}^{d 2}$, where the superscripts $p$ represent proximal and $d$ represent distal joints) and reference joint torques $\left(\tau_{d e s}^{p 1}, \tau_{d e s}^{d 1}\right.$, $\left.\tau_{\text {des }}^{p 2}, \tau_{\text {des }}^{d 2}\right)$. The feedback vector $\boldsymbol{y}$ has the same structure, but contains actual measured measured values. The desired joint angles (first half of the reference vector $\boldsymbol{u}$ ) are extracted by a feedforward matrix $\boldsymbol{F}$ and used as a feedforward term. The error between the reference $\boldsymbol{u}$ and feedback $\boldsymbol{y}$ is fed into a MIMO PI block (a combination of many P and PI controllers) which is a $4 \times 8$ matrix. The output of the PI block and the feedforward term are summed up as motor position command $\boldsymbol{c}$ (a $4 \times 1$ vector) and sent to the motors:

$$
\boldsymbol{c}(t)=\boldsymbol{F} \boldsymbol{u}(t)+\boldsymbol{K}_{p} \boldsymbol{e}(t)+\boldsymbol{K}_{i} \int_{0}^{t} \boldsymbol{e}(\tau) d \tau
$$

Here, $\boldsymbol{F}=\left[\begin{array}{ll}\boldsymbol{I}_{4 \times 4} & \boldsymbol{O}_{4 \times 4}\end{array}\right]$ is the feedforward matrix, $\boldsymbol{K}_{p}$ $(4 \times 8$ matrix with all entries being non-zero $)$ and $\boldsymbol{K}_{i}=$ $\left[\begin{array}{ll}\boldsymbol{K}_{4 \times 4} & \boldsymbol{O}_{4 \times 4}\end{array}\right]$ (where $\boldsymbol{K}$ is a matrix with all entries being non-zero) are proportional and integral gain matrices, and $e$ is the $8 \times 1$ error vector between $\boldsymbol{y}$ and $\boldsymbol{u}$. After that, the actual motor position vector $\boldsymbol{d}$ goes to the black-box system of the gripper and the unknown object. 
In the reference vector $\boldsymbol{u}$, the desired joint angle values $\left(\theta_{d e s}^{p 1}, \theta_{d e s}^{d 1}, \theta_{d e s}^{p 2}, \theta_{d e s}^{d 2}\right)$ are equal to those in the initial touch configuration, while the reference torques $\left(\tau_{d e s}^{p 1}, \tau_{d e s}^{d 1}, \tau_{d e s}^{p 2}\right.$, $\tau_{\text {des }}^{d 2}$ ) are chosen using the maximum motor torques. A special design of this controller is that we require the joint angles to be regulated exactly to the set points, but do not require the torques to be so. We allow and make use of the steady-state error of pure proportional control (we note that entries in the right half of the integral gain $\boldsymbol{K}_{i}$ are set to be zeros). In this way, the reference torques do not need to be a legal set of torques that result in equilibrium - actually, we are not able to design such a legal set of torques due to the absence of contact or object information. We let the law of dynamics decide the steady-state values for torques, and let the system balance itself automatically. The effectiveness of increasing joint torques is shown in section VI.

From a practical standpoint, the tuning process of the MIMO PI controller is not as complicated as it would seem based on the number of parameters. First, due to gripper symmetry, the number of parameters is cut by half. Second, we formulate every gain as a product of a baseline value $\left(b_{i}\right.$ in (3) (4) ) and a weight coefficient $\left(w_{i}\right.$ in (3) (4) ). The baseline values are set to be the same if the input entries corresponding to those gains have same physical dimensionality, and the weight coefficients are tuned based on its relative importance. Third, conventional tuning heuristics for the gains of single-input-single-output systems also apply here. The structures of the gain matrices are as follows:

$$
\begin{array}{r}
\boldsymbol{K}_{p}=\left[\begin{array}{llllllll}
w_{1} b_{1} & w_{2} b_{2} & w_{2} b_{1} & w_{2} b_{2} & w_{3} b_{3} & w_{4} b_{4} & w_{4} b_{3} & w_{4} b_{4} \\
w_{2} b_{1} & w_{1} b_{2} & w_{2} b_{1} & w_{2} b_{2} & w_{4} b_{3} & w_{3} b_{4} & w_{4} b_{3} & w_{4} b_{4} \\
w_{2} b_{1} & w_{2} b_{2} & w_{1} b_{1} & w_{2} b_{2} & w_{4} b_{3} & w_{4} b_{4} & w_{3} b_{3} & w_{4} b_{4} \\
w_{2} b_{1} & w_{2} b_{2} & w_{2} b_{1} & w_{1} b_{2} & w_{4} b_{3} & w_{4} b_{4} & w_{4} b_{3} & w_{3} b_{4}
\end{array}\right] \\
\boldsymbol{K}_{i}=\left[\begin{array}{lllllllll}
w_{1} b_{5} & w_{2} b_{6} & w_{2} b_{5} & w_{2} b_{6} & 0 & 0 & 0 & 0 \\
w_{2} b_{5} & w_{1} b_{6} & w_{2} b_{5} & w_{2} b_{6} & 0 & 0 & 0 & 0 \\
w_{2} b_{5} & w_{2} b_{6} & w_{1} b_{5} & w_{2} b_{6} & 0 & 0 & 0 & 0 \\
w_{2} b_{5} & w_{2} b_{6} & w_{2} b_{5} & w_{1} b_{6} & 0 & 0 & 0 & 0
\end{array}\right]
\end{array}
$$

We pick $b_{1}=0.2, b_{2}=0.5, b_{3}=4.0, b_{4}=8.0, b_{5}=1.0$, $b_{6}=1.0, w_{1}=1.0, w_{2}=0.3, w_{3}=1.0, w_{4}=0.5$ for our hardware.

\section{ENVELOPING GRASPING AND TRANSITIONS}

An enveloping grasp is the one where both distal and proximal links make contact with the object around its circumference. This type of grasp is generally considered more stable than a fingertip grasp because it can resist disturbances in a wider range of directions. The inability to envelop is also one of the main shortcomings of simple parallel grippers. In contrast, some of the more recent underactuated hands are optimized explicitly for effective enveloping grasps of a wide range of objects (e.g. [20]).

When using our proprioceptive gripper, we found that stable enveloping grasps for unknown objects are easier to obtain than fingertip grasps. The mechanism is generally fully constrained and all the links are counterbalancing each other. A simple joint torque control scheme, or the MIMO Grasping Controller, can fulfill this task.

A very important ability of this gripper, further underlining its versatility, is to transition between grasp types when holding unknown objects. After executing a stable fingertip grasp (using the MIMO Grasping Controller), the gripper can switch to joint torque control with the torque ratio (between distal and proximal joints) being 0.5 to 1.0 , thus bringing the object into the hand and creating an enveloping grasp. We illustrate this behavior with several experiments in the following section.

\section{EXPERIMENTS AND RESULTS}

In this section, we demonstrate the merits of the proprioception-enabled gripper by several experiments. We validated its capability of performing fingertip grasps, enveloping grasps, and the transition from the former to the latter. We note that in this study we only consider a twodimensional scenario, in which the objects are confined to move only in the plane of the fingers.

\section{A. Fingertip Grasp}

The goal of this experiment is to test the hypothesis that the MIMO Grasping Controller is effective in fingertip grasping for unknown objects. We compare against two baselines: a (fully-actuated) gripper running a Fixed Torque Ratio Controller, and a physical underactuated gripper.

The Fixed Torque Ratio Controller, where the torques applied to proximal and distal joints always follow a certain ratio, can be thought of as an emulation of a common type of underactuated grippers (tendon-pulley-driven, without special designs such as stoppers or clutches). When this kind of grippers make grasps, the configuration-dependent torques from the extension springs can be ignored, as they are usually much smaller than the flexing torques from the tendons. Therefore, the net joint torques in proximal and distal joint have a configuration-independent and design-time-fixed ratio, which is the ratio between the joint pulley radii.

Furthermore, we understand that this emulation is subject to limited control bandwidth and may have unrealistic behavior compared to its physical counterpart. Thus, we also built a physically underactuated gripper testbed for comparison. This testbed has same specs as the fully-actuated proprioceptive gripper, except that the proximal and distal joints are driven by a single tendon wrapping around the joint pulleys. In this design, we can alter the torque ratio by physically changing the pulleys between experiments. We perform torque control for proximal joints in our experiments, thus the torques on the distal joints are defined by the physically determined ratios. However, in this setup, we lose the ability to measure joint positions by SEA readings because, in underactuated mechanisms, joint positions are determined not only by actuator positions but also by contact forces which here are unknown.

1) Experiment Protocol: Our experiment proceeds as follows. We execute the grasping in two phases. In the first phase (approaching and touching), the fingers are set in torque control mode with very low reference torques so that they stop when they touch the object. In the second phase(squeezing), the gripper executes the MIMO Grasping 


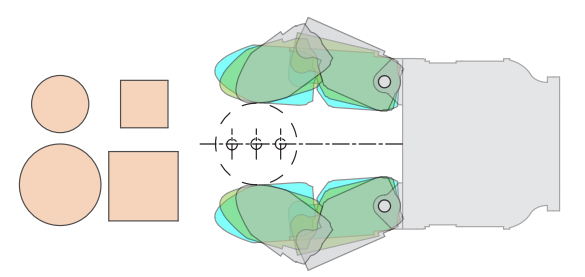

Fig. 5. Object sizes, object locations and initial touch poses.

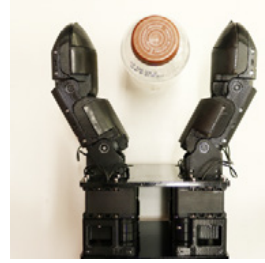

(a) Resting pose

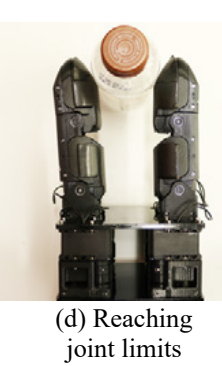

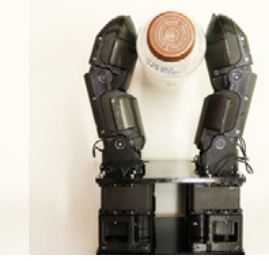

(b) Initial touch

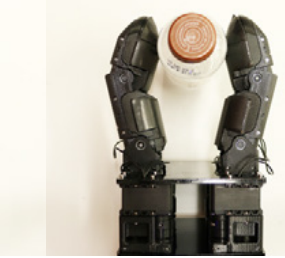

(c) Successful grasp near initial pose
Fig. 6. Pose changes in different controllers.

Controller or the Fixed Torque Ratio Controller in the fullyactuated testbed, or the joint torque control on proximal joints in the underactuated testbed for comparison.

There are a lot of factors that may influence the performance. To have a well-rounded comparison, we swept the following dimensions:

- Controllers. The torque ratio is a key parameter for both the Fixed Torque Ratio Controller, and the physically underactuated gripper. We tested the MIMO Grasping Controller against the other two baselines with three different ratios between the distal and proximal joint: $0.3,0.4$ and 0.5 .

- Objects. We selected four objects for the test: a big cylinder (diameter: 67mm), a big box (side length: $57 \mathrm{~mm}$ ), a small cylinder (diameter: $47 \mathrm{~mm}$ ) and a small box (side length: $39 \mathrm{~mm}$ ). All objects have negligible friction with the table.

- Object locations. We swept three locations along the center line of the gripper within the range of fingertip grasp: $100 \mathrm{~mm}, 120 \mathrm{~mm}$ and $140 \mathrm{~mm}$ from the palm.

- Initial touch poses. We tested three different distal joint angles for the initial touch: 0,30 and 60 degrees. We note that we only include this dimension for MIMO Grasping Controller and Fixed Torque Ratio Controller test, and not for the physically underactuated hand because the distal joint angles of initial touch cannot be explicitly controlled in runtime.

- Friction coefficient. We tested the controllers with two fingertip materials: rubber (high friction, $\mu=1.2$ ) and

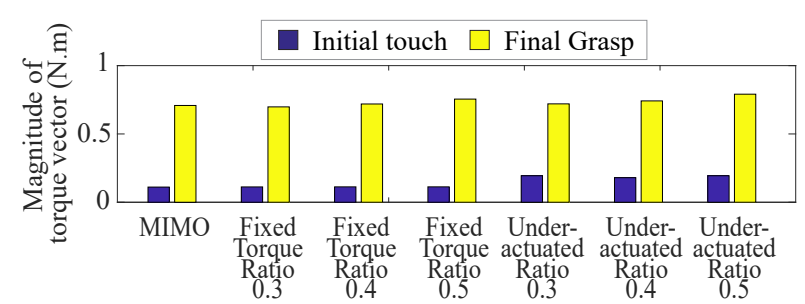

Fig. 7. Increases of torque magnitude during squeezing

vinyl plastic (low friction, $\mu=0.4$ ).

To sum up, we swept all five dimensions and conducted 360 grasping experiments. Fig. 5 shows the three object locations (shown as the crosshairs), three initial touch poses (colored fingers), and the sizes of the objects relative to the gripper (orange shapes).

\section{2) Performance Metric:}

- Success rate. The success rate is our primary performance metric. We define a "success" if the gripper finally settles down in equilibrium with the object in hand after squeezing. This definition includes three scenarios: (1) the gripper keeps the object in fingertips near initial touch pose, without converting to enveloping grasp or reaching joint limits, (2) the gripper holds the object but reconfigures to an enveloping grasp, and (3) the gripper keeps the object in fingertips but reaches a mechanical joint limit (thus the joint torque ratio changes). These cases are all considered successful but still need to be distinguished. Case (1) is the most desirable, while (2) and (3) mean the grasp is not stable at initial pose and relies on reconfiguration to be balanced.

- Gripper pose change. We believe it is also useful to keep the object in the same pose as when first contact is made. We thus use a secondary performance metric that evaluates how much the object moves in the hand during the squeezing process, with less movement considered better. Without access to object pose in Cartesian space, we measure this as the change in gripper pose between initial touch and final grasp (Euclidian distance in fourdimensional joint space). This metric is only calculated and averaged for the successful cases. Besides, it is not computed for physically underactuated gripper because the joint angles during grasping are not accessible for the reasons mentioned above.

3) Results: Fig. 6 shows the photos of some typical scenarios in the experiments. (a) and (b) shows the resting pose and the initial touch. (c) shows the successful grasp near initial touch pose. The other images show cases in which the object was kept in fingertip grasp but a joint limit was reached (d), the grasp was transformed into an enveloping one (e), and the object was squeezed out of the hand (f).

Fig. 7 shows that all controllers are effective in increasing joint torques. The horizontal axis shows different controllers (or grippers), the vertical axis is the magnitude of the fourdimensional torque vector $\left(\tau_{\text {meas }}^{p 1}, \tau_{\text {meas }}^{d 1}, \tau_{\text {meas }}^{p 2}, \tau_{\text {meas }}^{d 2}\right)$ which indicates how "strong" the grasp is, and bar colors 


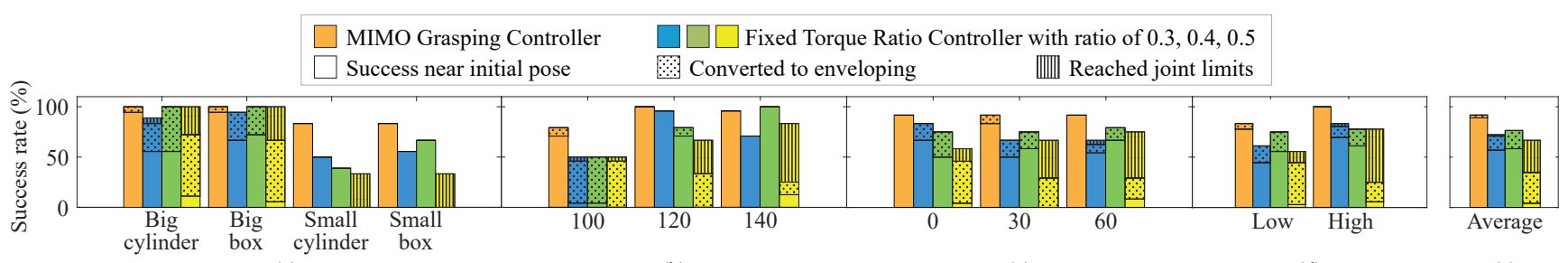

(a)

(b)

(c)

(d)

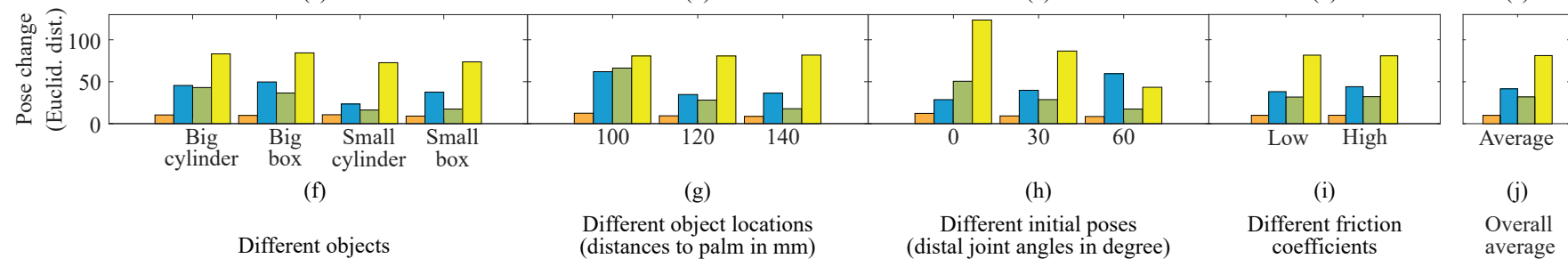

Fig. 8. Experiment results of fingertip grasping compared to Fixed Torque Ratio Controller.

distinguish between initial touch and final grasp. We can see that there is a significant increase in the torque magnitude, and the torque levels in different cases are similar.

The results of the experiments comparing against Fixed Torque Ratio Controller are visualized as multiple bar charts in Fig. 8 In each plot, the bar colors show four different controllers, the vertical axis is one of the performance metrics and the horizontal axis represents another dimension which is different in each plot (from (a) (f) to (d) (i): different objects, object locations, initial poses, and friction coefficients). In each bar in the first row showing the success rate, the purecolor area, the dotted area, and the line-shaded area represent, respectively, successful fingertip grasp without reaching joint limit, successful grasps but converted to enveloping, as well as successful fingertip grasp but joint angles reached limits.

Similarly, the results comparing against physically underactuated grippers are shown in Fig. 9 Here, the initial touch pose dimension is not available, so there are three dimensions (from (a) to (c): different objects, object locations, and friction coefficients). Also, the pose change metric is not available due to the absence of joint angle information. All other plotting rules are the same as Fig. 8 ,

As shown in Fig. 8 (e)(j) and Fig. 9 (d), the overall success rates are $91.67 \%$ for MIMO Grasping Controller, $72.22 \%, 76.39 \%$, 66.67\% for Fixed Torque Ratio Controller with torque ratio of $0.3,0.4,0.5$, respectively, and $87.50 \%$, $75.00 \%, 87.50 \%$ for physically underactuated gripper with torque ratio of $0.3,0.4,0.5$, respectively. Even when the overall success rates are close (for example, Fig. 9 (d)), the types of the resulting grasps are significantly different. Besides, the gripper pose change metric for the MIMO controller is 9.96 , compared to $41.55,31.93$, and 81.14 (degrees) respectively for the Fixed Torque Ratio controllers.

\section{B. Enveloping Grasps and Transitions}

We performed a second experiment to show that this gripper can perform enveloping grasp with either MIMO Grasping Controller or Fixed Torque Ratio Controller. We tested on two objects (big cylinder and big box), two object locations (60mm and $80 \mathrm{~mm}$ from the palm), and two controllers mentioned above. The success rate is $100 \%$.

The last experiment is to show the performance of the transition from fingertip grasp to enveloping grasp. We first created fingertip grasps using the MIMO Grasping Controller, and then switched to Fixed Torque Ratio Controller with a ratio of 0.5 . We tested on two objects (big cylinder and big box), three object locations (100, 120 and 140mm), three initial poses ( 0,30 and 60 degrees) with the low friction fingertips. We found the success rate was $83.33 \%$.

\section{Discussion AND CONCLUSION}

Overall, the results of the previous section support our hypotheses: the proprioceptive gripper running MIMO Grasping Controller is effective at executing stable fingertip grasps in a variety of situations and outperforms the baselines. Furthermore, the proprioceptive gripper exhibits versatility in being able to perform multiple types of grasps and also to transition between them on-demand.

Based on Fig. 8 and 9, the MIMO Grasping Controller outperforms the baselines in fingertip grasping, and usually succeeds without transforming to an enveloping grasp or reaching joint limits. In contrast, the emulated and physical underactuated gripper often transform to an enveloping grasp or reach joint limits, thus relying on gripper reconfiguration. The second row of Fig. 8 ((f) to (j)) gives similar intuition: for the Fixed Torque Ratio Controller, most cases have a large pose change. While the end-result is stable, it is different from the originally intended grasp. This might be unimportant or detrimental depending on the application.

It is also interesting to notice that, in different conditions, the optimal torque ratio for the emulated or physical underactuated gripper is different. We take this to mean that there is no one clearly preferable pre-set torque ratio, which could be physically implemented in a mechanical design, in order to obtain ideal performance in all these cases. In contrast, the proprioception-enabled gripper has the flexibility to alter torques at run-time.

Looking at how specific variables affect performance we can gain additional insights. From Fig. 8 and 9 (a) and (b) we can see that the success rates for Fixed Torque Ratio 


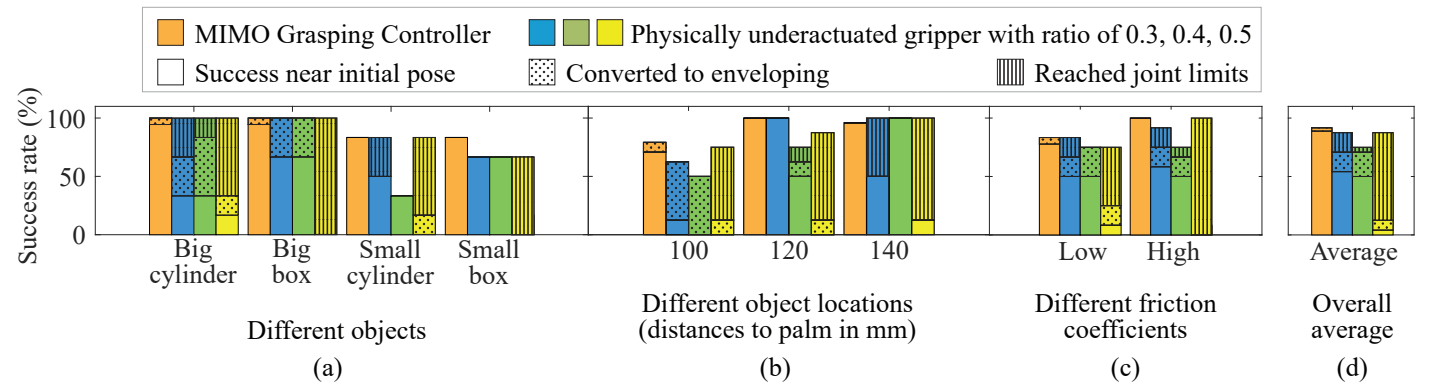

Fig. 9. Experiment results of fingertip grasping compared to physically underactuated gripper.

Controller are low if the objects are small and close to the palm. This is because the emulated or physical underactuated gripper tends to transform the initial unstable fingertip grasps to enveloping when contacts are close to distal joints, but cannot cage the object if it is small because the distal links are fighting against each other - a common issue for underactuated grippers. In contrast, the MIMO Grasping Controller does not suffer from this because it does not perform the conversion.

In the transitioning experiment, the high success rate shows the proprioceptive gripper can indeed perform the conversion between grasp types on-demand. Though underactuated grippers also occasionally perform such transitions, they occur unintentionally and without giving the user an option to select the desired type of grasp.

It is important to also highlight the limitations of this study. Due to high dimensionality of the brute-force sweep in our experiment, we cannot afford to cover a larger range with a finer resolution for each dimension. In particular, we are unable to explore more possibilities for physically implemented torque ratios. The evaluation of the controller is primarily experimental and would benefit from additional stability analysis, carried out for example for representative cases and grasps.

Overall, we claim that proprioceptive manipulators, using active sensing and control such as the MIMO Grasping Controller, represent a promising way towards more versatile grasping and manipulation for unknown objects. Future work will include the extension of the operation to threedimensional cases, optimization / learning of the control gains, and the inclusion of hand position to our set of actively controlled variables. We are aiming to further explore these possibilities.

\section{REFERENCES}

[1] C. Blanchard, R. Roll, J.-P. Roll, and A. Kavounoudias, "Differential contributions of vision, touch and muscle proprioception to the coding of hand movements," PloS one, vol. 8, no. 4, p. e62475, 2013.

[2] T. Yoshikawa, "Control algorithm for grasping and manipulation by multifingered robot hands using virtual truss model representation of internal force," in IEEE Intl. Conf. on Robotics and Automation, vol. 1, 2000, pp. 369-376.

[3] S. Arimoto, P. T. A. Nguyen, H.-Y. Han, and Z. Doulgeri, "Dynamics and control of a set of dual fingers with soft tips," Robotica, vol. 18, no. 01, pp. 71-80, 2000.

[4] F. Caccavale, G. Muscio, and F. Pierri, "Grasp force and object impedance control for arm/hand systems," in 16th International Conference on Advanced Robotics (ICAR), 2013, pp. 1-6.
[5] S. A. Schneider and R. H. Cannon, "Object impedance control for cooperative manipulation: Theory and experimental results," IEEE Transactions on Robotics and Automation, vol. 8, no. 3, pp. 383-394, 1992.

[6] S. Arimoto, R. Ozawa, and M. Yoshida, "Two-dimensional stable blind grasping under the gravity effect," in IEEE Intl. Conf. on Robotics and Automation (ICRA). IEEE, 2005, pp. 1196-1202.

[7] M. Yoshida, S. Arimoto, and J.-H. Bae, "Blind grasp and manipulation of a rigid object by a pair of robot fingers with soft tips," in IEEE Intl. Conf. on Robotics and Automation (ICRA). IEEE, 2007, pp. 4707-4714.

[8] D. Wang, B. T. Watson, and A. H. Fagg, "A switching control approach to haptic exploration for quality grasps," Robotic Science and Systems, 2007.

[9] R. Platt Jr, A. H. Fagg, and R. A. Grupen, "Null-space grasp control: Theory and experiments," IEEE Transactions on Robotics, vol. 26, no. 2, pp. 282-295, 2010.

[10] K. Hsiao, S. Chitta, M. Ciocarlie, and E. G. Jones, "Contact-reactive grasping of objects with partial shape information," in IEEE/RSJ Intl. Conf. on Intelligent Robots and Systems (IROS), 2010, pp. 1228-1235.

[11] C. Lovchik and M. A. Diftler, "The robonaut hand: A dexterous robot hand for space," in IEEE Intl. Conf. on Robotics and Automation (ICRA), vol. 2, 1999, pp. 907-912.

[12] J. Butterfaß, M. Grebenstein, H. Liu, and G. Hirzinger, "Dlr-hand ii: Next generation of a dextrous robot hand," in IEEE Intl. Conf. on Robotics and Automation (ICRA), vol. 1, 2001, pp. 109-114.

[13] A. Edsinger-Gonzales and J. Weber, "Domo: A force sensing humanoid robot for manipulation research," in 4th IEEE/RAS Intl. Conf. on Humanoid Robots, vol. 1, 2004, pp. 273-291.

[14] E. Torres-Jara, "Obrero: A platform for sensitive manipulation," in 5th IEEE/RAS Intl. Conf. on Humanoid Robots, 2005, pp. 327-332.

[15] G. A. Pratt and M. M. Williamson, "Series elastic actuators," in IEEE/RSJ Intl. Conf. on Intelligent Robots and Systems (IROS), vol. 1, 1995, pp. 399-406.

[16] M. Grebenstein, A. Albu-Schäffer, T. Bahls, M. Chalon, O. Eiberger, W. Friedl, R. Gruber, S. Haddadin, U. Hagn, R. Haslinger, et al., "The dlr hand arm system," in IEEE Intl. Conf. on Robotics and Automation (ICRA), 2011, pp. 3175-3182.

[17] A. M. Dollar and R. D. Howe, "The highly adaptive sdm hand: Design and performance evaluation," The International Journal of Robotics Research, vol. 29, no. 5, pp. 585-597, 2010.

[18] L. U. Odhner, L. P. Jentoft, M. R. Claffee, N. Corson, Y. Tenzer, R. R. Ma, M. Buehler, R. Kohout, R. D. Howe, and A. M. Dollar, "A compliant, underactuated hand for robust manipulation," The International Journal of Robotics Research, vol. 33, no. 5, pp. 736-752, 2014.

[19] L. Birglen and C. M. Gosselin, "Kinetostatic analysis of underactuated fingers," IEEE Transactions on Robotics and Automation, vol. 20, no. 2, pp. 211-221, 2004.

[20] M. Ciocarlie, F. M. Hicks, R. Holmberg, J. Hawke, M. Schlicht, J. Gee, S. Stanford, and R. Bahadur, "The velo gripper: A versatile single-actuator design for enveloping, parallel and fingertip grasps," The International Journal of Robotics Research, vol. 33, no. 5, pp. 753-767, 2014.

[21] S. Ates, V. I. Sluiter, P. Lammertse, and A. H. Stienen, "Servosea concept: cheap, miniature series-elastic actuators for orthotic, prosthetic and robotic hands," in 5th IEEE RAS \& EMBS Intl. Conf. on Biomedical Robotics and Biomechatronics, 2014, pp. 752-757. 